\title{
Study Personnel Effective Date Range
}

National Cancer Institute

\section{Source}

National Cancer Institute. Study Personnel Effective Date Range. NCI Thesaurus. Code C94100.

The date and time span for when the study site personnel is active. 